\title{
Intra- and Periarticular Ganglia (Synovial Cysts) of the Hip with Compression of the Obturator Nerve, Concomitant with Lesions of the Ligamentum Teres-A Report of 3 Cases
}

\author{
Florian Haug*, Richard Herzog \\ Investigation performed at Department of Orthopedics and Trauma Surgery, Lucerne Cantonal Hospital, Wolhusen, Switzerland \\ Email: *florian.haug@luks.ch
}

How to cite this paper: Haug, F. and Herzog, R. (2021) Intra- and Periarticular Ganglia (Synovial Cysts) of the Hip with Compression of the Obturator Nerve, Concomitant with Lesions of the Ligamentum Teres-A Report of 3 Cases. Open Journal of Orthopedics, 11, 170-182. https://doi.org/10.4236/ojo.2021.115017

Received: March 12, 2021

Accepted: May 28, 2021

Published: May 31, 2021

Copyright (c) 2021 by author(s) and Scientific Research Publishing Inc. This work is licensed under the Creative Commons Attribution International License (CC BY 4.0).

http://creativecommons.org/licenses/by/4.0/ (c) (i) Open Access

\begin{abstract}
Background: Synovial cysts of the hip are commonly found in patients with intra- or extraarticular pathologies of the joint. Symptoms are mostly unspecific. To date there are no guidelines for a gold standard of treatment. Aim of this article is to show up how lesions of the ligamentum teres (LT) might possibly lead to a specific formation of synovial cysts of the hip joint and how this can be treated arthroscopically. Methods: This case series included 3 patients with ganglia of the hip. All patients had impingement symptoms, combined with untypical location of pain. All patients qualified for joint preserving surgery and underwent hip arthroscopy with pre- and postoperative MRI imaging. The mean follow-up time was 22 months. Results: MRI imaging showed extensive ganglia, presumably originating from the pelvic root of LT, extending to the obturator lodge. In 2 of 3 cases MRI showed lesions of the LT. Hip arthroscopy revealed damage of the LT in all cases, caused by chronic instability of the joint. The postoperative MRI showed a complete regression of the ganglia in all patients after offset correction. After follow-up, 2 of 3 patients were mostly symptom free. One patient was still suffering from a chronic weakness of the gluteus medius muscle. Conclusion: Whenever unspecific radiating pain of surrounding areas of the hip is encountered and cannot be explained by common pathologies of the hip, possible compression of nerves by ganglion cysts should be excluded. This should be done by MRI arthrography. A partial rupture of the LT can occur during FAI with consecutive formation of ganglia in the obturator canal, compressing the obturator nerve. Primarily the articular pathology needs to be repaired. In our cases,
\end{abstract}


this was feasible by hip arthroscopy, as a minimally invasive and safe technique.

\section{Keywords}

Ganglion of the Hip, Lesion of Ligamentum teres, Hip Arthroscopy

\section{Introduction}

Synovial cysts are commonly found in the shoulder or the knee joint. The exact cause is still unknown, however the most common thesis is an excessive production of synovial fluid due to an intraarticular pathology [1] [2]. Apart from necrosis and trauma, these involve osteoarthritis, rheumatoid arthritis and labral tears. In some cases cysts might be caused by inflammation of extraarticular bursas [3].

Most synovial cysts of the hip are located anterosuperiorly. The bursa of the iliopsoas communicates with the hip joint in $15 \%$ of the cases. In patients with osteoarthritis this is even more common and can be found in up to $40 \%$. The bursa lies between the iliofemoral and the pubofemoral ligament, where the joint capsule has its thinnest portion [4]. This might explain why anterosuperior cysts of the hip joint are more common than posterior cysts.

Posteromedial cysts, mostly protrude between the ischiofemoral ligament and the zona orbicularis, compressing the obturator nerve [5]. Paralabral cysts are formed when synovial fluid passes paralabral tears through a check valve mechanism [6]. Labral tears are mostly found in the anterosuperior labrum. Accordingly, $56 \%$ of the paralabral cysts are located anterosuperiorly, whereas only $17 \%$ are located posteriorly [7].

The Ligamentum teres originates at the transverse acetabular ligament and the pubic and ischial margins of the acetabular notch. The function of the Ligamentum teres (LT) has not been fully clarified to date. According to recent studies, it restricts external rotation and flexion in the hip joint, where it acts as a final stabilizer [8] [9] [10]. Additionally, the LT seems to have mechanoreceptors which help to counter micro instability by activating muscular stabilizers of the hip joint [11]. Consequently, chronic instability, such as dysplasia or femoroacetabular impingement (FAI) with hypomochlion effect, can lead to recurrent overstretching of the LT and concomitant lesions [12]. This can also be caused by certain sports where a large range of motion is required [10].

\section{Symptoms, Signs and Diagnostics}

Lesions of the LT and synovial ganglia of the hip joint are both, often asymptomatic and unspecific [1] [2], as symptoms and signs are mostly superimposed by underlying pathologies of the hip joint. Partial lesions of the LT often manifest as hip or groin pain, along with locking or a sensation of instability [9]. In Physical examination, patients with lesions of the LT often present with a positive 
anterior impingement sign. In recent studies, asymptomatic cystic lesions of the hip were found in 26 out of 129 (20.2\%) MRIs [13] [14]. Synovial cysts might become symptomatic when compressing adjacent structures, such as nerves or vessels [2].

\section{Compression of peripheral nerves}

As synovial cysts are mostly located anteriorly of the hip joint, symptoms are mostly caused by compression of the femoral nerve. To date, 22 of such cases are described in literature. Patients describe tenderness over the anterior hip and radiating pain, down the medial thigh over the knee, down to the medial side of the foot, mimicking L2-4 radiculopathy [2]. Joint related cysts, compressing the obturator nerve, are to date only described in 8 cases [15]-[22]. Patients described tenderness of the adductor muscles as well as pain of the groin and the anteromedial thigh. In chronic cases weakness of the adductor muscles was described. Other cases describe compression of the sciatic nerve, with typical sciatic pain, radiating from the buttock, down the posterior thigh, to the lateral aspect of the lower leg. Compression of arteries is associated with intermittent claudicatio and coolness of the foot, whereas compression of veins where described in 40 cases, resulting in swelling of the leg [2].

\section{Pathophysiology}

The underlying cause varies for each case, although they all have an intraarticular pathology in common. Those included anterior labral tears and osteoarthrosis of the hip [20]. In one case the stalk of the cyst could be followed to the anterior labrum [16], whereas in another case the stalk was following the obturator externus muscle [15]. Ganglion cysts arising from the transverse ligament have been described in 3 cases [3] [19], although reviews have shown, that they might as well originate from articular branches of the obturator nerve, proceeding an intraneural path [21].

The obturator nerve follows the linea terminalis, lying behind and medial of the psoas muscle. Below the linea terminalis it runs through the obturator foramen at its anterolateral border. Here it splits into an anterior and a posterior branch, divided by the adductor brevis muscle. Its motor nerves innervate the adductor muscles, where its sensitive nerve innervates the distal part of the inner thigh (Figure 1).

\section{Treatment}

\section{Synovial cysts of the hip}

To date there is no gold standard in treatment of symptomatic synovial cysts of the hip. In case of asymptomatic synovial cysts, observation is possible. Conservative therapy or other approaches like synovectomy did not show any significant improvement of symptoms [23]. Infiltration has shown to reduce the symptoms [2] [19]. Yukata et al. suggests infiltration as a possible first line therapy for symptomatic relief. Although, 37\%, recurrence has been reported, compared to surgery, with only $5 \%$ recurrence [24]. With surgery, correction of the 


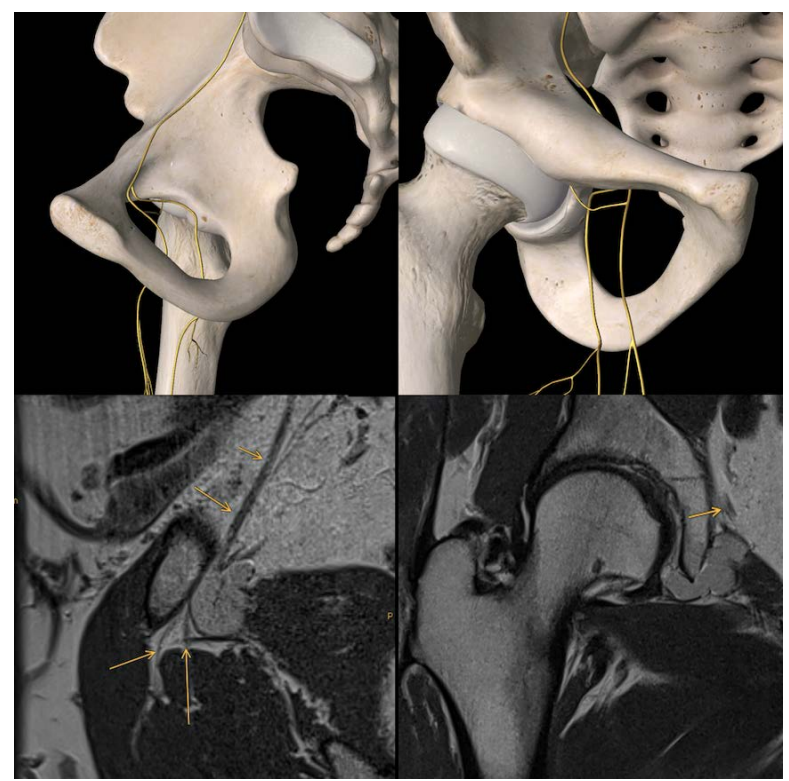

Figure 1. Transverse and frontal view of the obturator nerve, passing through the obturator foramen. More distally the anterior and posterior branches are indicated. The MRI images were taken from one of our cases, where the proximity to the cyst can be seen. Source upper left and right: Essential Anatomy 5 (by 3D4Medical from Elsevier), lower left and right: Case Nr. 2)

underlying intraarticular pathology is possible. Therefore surgery seems to be the only curative option [1] [21].

\section{Lesions of the $L T$}

Recent literature reviews reported good outcomes after debridement of the LT. Pergaminelis et al. treated 35 Patients with isolated lesions of the LT with arthroscopic radiofrequency ablation [23]. They reported significant improvement of the symptoms for a mean period of 17.7 months (range 6 - 42 months). In some cases, a persisting sensation of instability was reported. Those might qualify for reconstruction of the LT, although the benefit of LT-reconstruction remains unclear. Various possibilities, with different graft sources have been described in a few case reports and some small case studies [9] [10] [11].

\section{Case Reports}

\subsection{Case, Mr. F, 43 y/o}

A 43-year-old male patient presented with typical impingement complaints of the right hip, mostly on exertion. The initial MRI confirmed an offset disorder with small chondrolabral tearing and extensive ganglia, originating from a mucoid degenerated, thinned out LT. These extended beyond the transverse ligament into the obturator foramen (Figure 2(a), Figure 2(b)). Intraoperatively, it was shown that one of the ganglia originated directly from the partially ruptured LT (Figure 2(c)). The ligament and the intra-articular part of the ganglia were resected, causing the ganglia to empty (Figure 2(d), Figure 2(e)). Additionally, the 


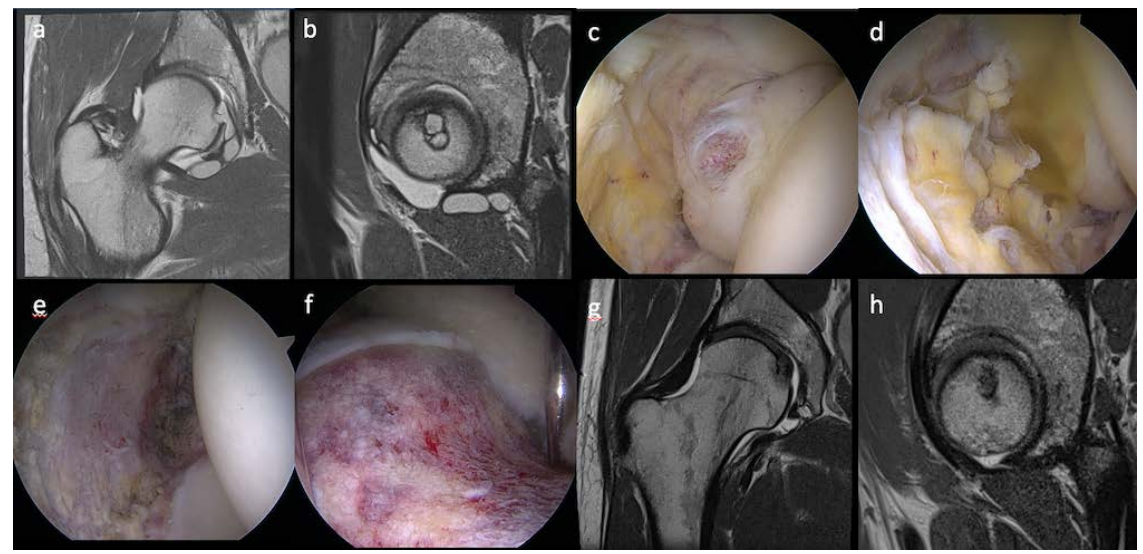

Figure 2. (a) ap. and (b) transversal preoperative MRI, (c) intraoperative image of partially ruptured LT with cyst, (d) cyst after incision, (e) acetabular fossa after resection of the LT, (f) offset correction, (g) ap and (h) transversal postoperative MRI after 3 months.

offset was corrected (Figure 2(f)). The patient quickly became mostly symptom-free. Due to renewed complaints, a check-up with a control MRI was carried out, which showed a good correction of the offset (Figure 2(g), Figure $2(\mathrm{~h})$ ). The original edema in the anterolateral labrum had disappeared, however a small capsule weakness, adhesions and remaining chondrolabral damage could be seen. The ganglia have receded completely, although only a partial resection was performed.

\subsection{Case, Mr. S, 43 y/o}

A 43-year-old male patient presented with chronic pain of the left hip since 1 1/2 years. The pain was located mostly gluteal and lateral of the hip, radiating to the medial thigh with a burning sensation. The patient works in retail trade. At the time of presentation, he was $100 \%$ unfit for work. MRI showed a synovial cyst of the hip, extending to the obturator loge, compressing the obturator nerve, as well as a slight offset disorder (Figure 3(c)). Conservative treatment like analgesics, physiotherapy or infiltration of the sacroiliac joint showed no relief. Puncture of the joint ganglion brought only short-term improvement. Although the MRI did not show any significant joint pathology responsible for the complaints, a hip arthroscopy was performed. It showed an edema of the labrum and the LT (Figure 3(a), Figure 3(b), Figure 3(e)). The ligament was resected and the offset corrected. There were no intraarticular ganglia visible. The radiating pain to the medial thigh quickly disappeared. The remaining symptoms are presumably caused by muscle weakness of the gluteus medius and the piriformis muscles and improved over time. 3 months postoperative an MRI was performed, which confirmed complete regression of the ganglia that had compressed the obturator nerve (Figure $3(\mathrm{~d})$ ). 3 months postoperatively the patient was able to take up his work to $100 \%$.

\subsection{Case, Mrs. R, 47 y/o}

A 47-year-old female patient was suffering from chronic recurrent perineal pain 


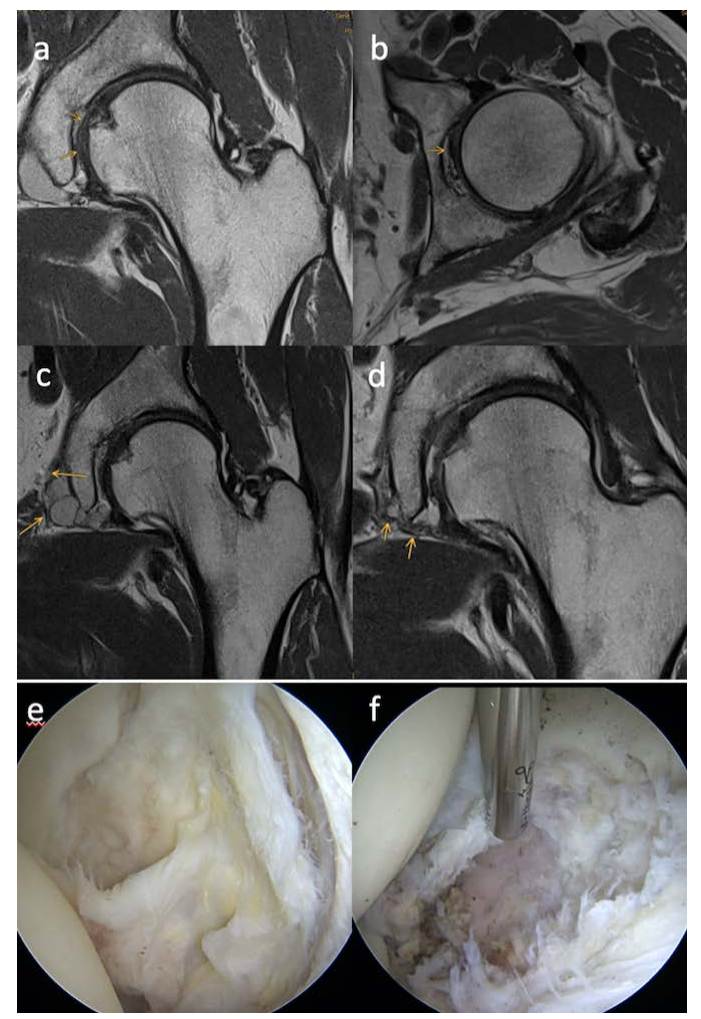

Figure 3. (a) ap. and (b) axial preoperative MRI, the arrows indicating the LT, with the origin of the cysts at its base (c) preoparative MRI, showing the proximity of the obturator nerve to the cysts (d) postoperative MRI after 3 months (e) intraoperative images of the partially ruptured LT (f) acetabular fossa after resection of the LT.

for 5 years. The pain was exercise-related pain, also induced by long sitting. It was described as radiating pain to the genital area, with a burning sensation. 3 years ago, after visiting different specialists a uterus myomatosus was diagnosed and a total hysterectomy was performed. This didn't show any relief. Also, medication with Nsaids and tricyclic antidepressants didn't help. She used to be physically active, whereas due to the current situation she now focuses on Pilates and physiotherapy. Still, the pain forced her to reduce work to $80 \%$. Native MRI showed small ganglia of the hip joint, whereas MR arthrography showed extensive ganglia, reaching from the hip joint to the obturator loge, as well as a slight cam impingement (Figure 4). Assuming a pathology of the LT we performed an arthroscopy of the hip to resolve the intraarticular pathology. Extraarticular preparation below the psoas muscle allowed visualization of the ganglion through the obturator foramen, where the cyst could be opened (Figures 5(a)-(c)). Intraarticular the slight offset disorder was confirmed, and an edema of the Labrum could be seen. The offset disorder was corrected (Figure 5(f)). The LT showed a chronic partial rupture (Figure 5(d)). After resection of the LT another cyst could be visualized and drained in the medial part of the acetabular fossa (Figure 5(e)). Postoperatively the patient reported a sensation of relieved pressure in the perineal and genital area. 3 months postoperatively the exercise-related pain 
was gone. The burning sensation returned after stopping her tricyclic antidepressants. The follow-up MRI showed complete regression of the ganglia.

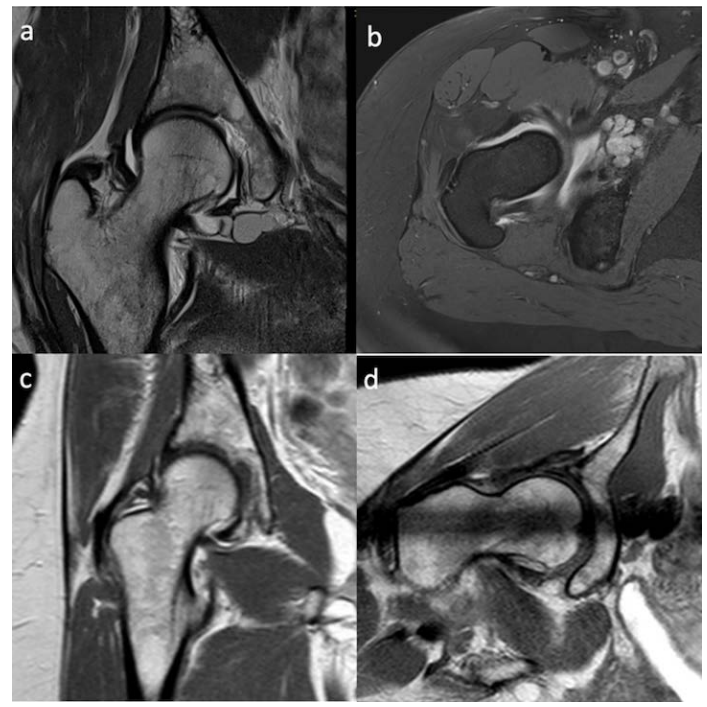

Figure 4. (a) ap. and (b) axial preoperative MRI with extensive cysts (c), (d) MRI 3 months after surgery. The cysts have disappeared.

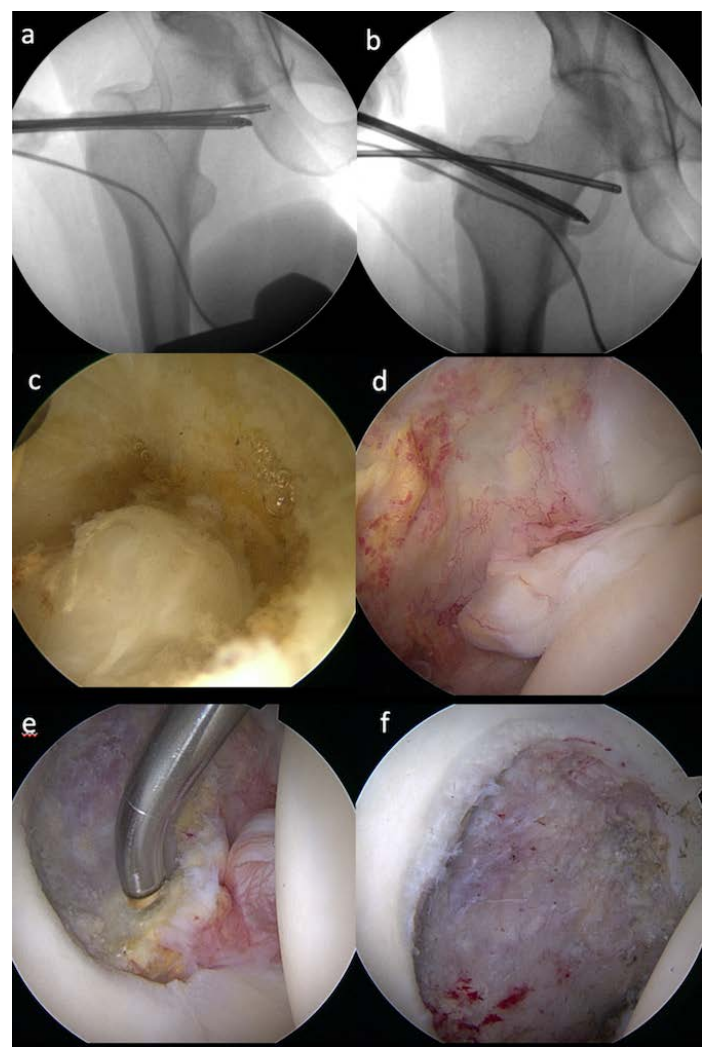

Figure 5. (a) - (c) Arthroscopic approach to the obturator foramen. Intraoperative imaging with $\mathrm{x}$-ray and arthroscopic visualization. (c) extraarticular view of the cyst, protruding through the obturator foramen (d) intraoperative image of partially ruptured LT with signs of inflamation, (e) acetabular fossa after resection of the LT, (f) offset correction. 


\section{Discussion}

\section{Symptoms}

In our cases the pain was mostly located in different areas around the hip. Most radicular pain that is caused by an intraspinal source is greater in the leg than in the back [25]. The symptoms are therefore more suggestive for an intraarticular pathology of the hip, rather than a spinal genesis. Table 1, suggests a typical presentation of chronic recurrent pain with a burning sensation, radiating to the medial thigh or to the genitals. This was observed if the obturator nerve was compressed by the ganglia. (The radiating pain to the genitals might be explained by compression of the external pudendal artery and vein.) All patients described worsening of the symptoms during physical exertion, although resting pain was common, especially after long sitting. The painful episodes often improved after long term physical rest. 2 of 3 Patients reported chronic pain, resulting in significant limitations in work and daily activities.

\section{Clinical findings}

The clinical findings seen in Table 2 were unspecific. One patient had an impaired internal rotation and a positive anterior impingement sign. Other than that, the range of motion was within normal limits. There was no significant loss in muscle strength.

\section{Radiological findings}

As shown in Table 3 conventional radiographs did not show any significant pathology, explaining the symptoms mentioned above. They all had a mild cam deformity. MR Arthrography showed lesions of the LT in 2 of 3 Patients and the above-mentioned ganglia, which were all protruding through the obturator foramen. A review from Hafezi-nejad et al. showed a sensitivity of $82.2 \%$ and specificity of $88.6 \%$ of MR Arthrography in detecting lesions of the LT, compared to a significantly lower sensitivity in native MRI (64.7\%) [26]. Consequently, lesions of the LT can only be safely excluded by hip arthroscopy.

Table 1. Presenting complaint.

\begin{tabular}{ccccc}
\hline & & Mr. F. & Mr. S. & Mrs. R. \\
\hline Gluteal pain & 1 & 0 & 1 & 0 \\
Thigh pain & 1 & 0 & 1 & 0 \\
Inguinal pain & 1 & 1 & 0 & 0 \\
Perineal pain & 1 & 0 & 0 & 1 \\
Genital pain & 2 & 0 & 1 & 1 \\
Pain on exertion & 3 & 1 & 1 & 1 \\
Pain after long standing & 1 & 0 & 1 & 0 \\
Pain after long sitting & 3 & 1 & 1 & 1 \\
Pain w/o exertion & 2 & 0 & 1 & 1 \\
Night pain & 1 & 0 & 1 & 0 \\
Burning sensation & 3 & 1 & 1 & 1 \\
Radiating pain & 2 & 0 & 1 & 1 \\
\hline
\end{tabular}


Table 2. Clinical findings.

\begin{tabular}{cccc}
\hline & Mr. F. & Mr. S. & Mrs. R. \\
\hline Reduced range of motion & 1 & 0 & 0 \\
Flexion/Extension path & $100 / 0 / 20^{\circ}$ & $110 / 0 / 5^{\circ}$ & $110 / 0 / 20^{\circ}$ \\
Flexion/Extension phys & $115 / 0 / 20^{\circ}$ & $115 / 0 / 10^{\circ}$ & \\
ext./int. Rotation path & $40 / 0 / 0^{\circ}$ & $35 / 0 / 30^{\circ}$ & $50 / 0 / 35^{\circ}$ \\
ext./int. Rotation phys & $40 / 0 / 25^{\circ}$ & $35 / 0 / 35^{\circ}$ & \\
Ab-/Adduction path & $45 / 0 / 30^{\circ}$ & & $45 / 0 / 30^{\circ}$ \\
Ab-/Adduction phys & $45 / 0 / 30^{\circ}$ & & \\
Impingement test positive & 1 & 0 & 0 \\
\hline
\end{tabular}

Table 3. Radiologic findings.

\begin{tabular}{cccc}
\hline & Mr. F. & Mr. S. & Mrs. R. \\
\hline Cam deformity & 1 & 1 & 1 \\
Alpha Angle & 51 & 72 & 70 \\
Antetorsion & 6 & 128 & 26 \\
CCD Angle & 123 & 32 & 145 \\
CE Angle & 29 & & 35 \\
Labrum & 1 & 0 & 1 \\
Tear & 1 & 0 & 1 \\
Degeneration & & & \\
Cartilage & 0 & 0 & 0 \\
Tear & 1 & 0 & 0 \\
Degeneration & edema & edema & 0 \\
Lig. teres & 1 & & 0 \\
Acet. retroversion & $64 \times 9$ mm & $2.5 \times 17 \times 12 \mathrm{~mm}$ & $33 \times 28 \times 15 \mathrm{~mm}$ \\
Ganglia & 0 & 0 & 0 \\
Crossover sign & 0 & 0 & 0 \\
Posterior wall sign & & & \\
\hline
\end{tabular}

\section{Pathophysiology}

Briem et al. suggests that recurrent sustained micro-subluxations of the hip joint, due to bony deformities might cause capsular stress [25]. We believe, that in our cases, the offset and pincer disorders or rotational disorders, as primary pathology, lead to subluxations of the hip, due to a hypomochlion effect. This results in strain on the LT and the labrum with concomitant lesions. This leads to extensive production of synovial fluid. With increased intraarticular pressure, the synovial fluid may enter der LT through those microlesions, causing the ganglia to grow in size. After physical rest the excess synovial fluid is partially resorbed. The compressing effect of the ganglia on adjacent structures decreases. Therefore, pain on exertion might possibly be associated with a fluctuating size of the ganglia. In the presented cases, excessive production of synovial fluid 
might also be caused by stress on the labrum due to mild FAI.

\section{Intraoperative findings}

Arthroscopically a chronic lesion of the LT was seen in all three cases, as well as edema of the labrum. CAM disorders were mild, and the labrum didn't require reconstruction. The ganglion cysts were directly associated with the LT in 2 out of 3 cases and could directly be drained in 2 out of 3 cases. In one case the ganglion could be drained directly from the LT. In the other case, the stalk of the cysts was found directly medially of the LT. This supports our suggestion.

\section{Treatment}

The presented cases had minor pathologies of the hip joint in common, which, if asymptomatic, wouldn't qualify for surgery. In case number 2 and 3 conservative treatments like physiotherapy and analgesics didn't show any relief. Hip arthroscopy is a safe method to address the intraarticular pathologies. In all 3 cases, this led to an acute relief of symptoms. Extraarticular the approach to the obturator foramen seems a good way to reach the ganglia. The intraarticular part seems to be addressed best in the acetabular fossa. Here the LT originates in the very medial part of the fossa, just posterior of the transverse ligament. After resection of the LT, the stalk of the cyst can be visualized and drained.

Follow-up images showed continuous regression of the ganglia. The long-term follow-up MRI images showed regression of the cysts, even if they weren't opened intraoperatively.

Preoperative aspiration of the cysts would have been a possible option. This has to be discussed with each patient individually, pointing out clearly, that this doesn't address the primary problem and recurrence is likely. An open approach, via surgical subluxation of the hip was described in a similar case, where a synovial ganglion of the hip was thought to be causing L5 radiculopathy [25]. This is a possible option, although, in our opinion there is no benefit in open surgery.

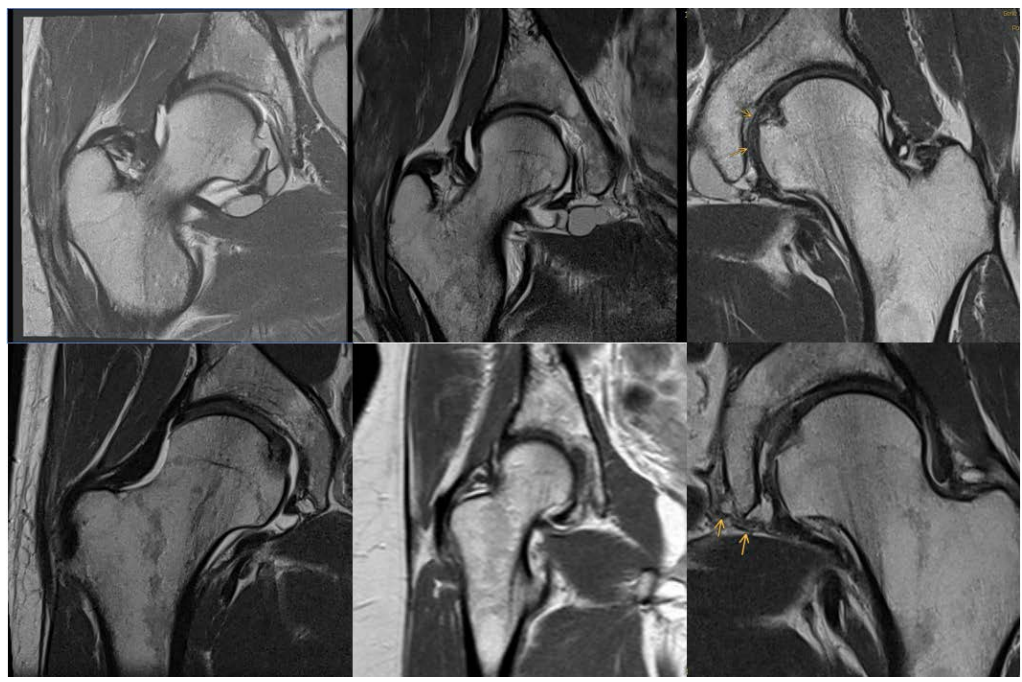

Figure 6. Preoperative (first row) and postoperative (second row) images, showing complete regression of the ganglia in all cases (case number $1-3$ from left to right). 


\section{Conclusions}

The above-mentioned cases show that a partial rupture of the LT and formation of ganglia can occur during hip impingement. This may cause unspecific neurological symptoms and untypical pain. Our findings were suggestive for microlesions of the LT causing the formation of synovial cysts. Although, the exact cause remains uncertain. In other cases intermittent claudicatio or leg edema, have been described [2]. Those may easily be misinterpreted as vascular or lumbar pathologies. When in doubt we suggest performance of an MR arthrography in addition to diagnostics of lumbar or vascular disease. This might reveal ganglia formation around the hip joint, although lesions of the LT might be missed [23] [26]. In analogy to the Baker cyst of the knee, primarily the articular damage needs to be repaired to achieve permanent relief. In our cases, this included resection of the damaged LT and, if possible, resection of the ganglia, as well as correction of the offset. For this purpose, hip arthroscopy is a minimally invasive and safe technique (Figure 6). Due to the incision site being further away from the neurovascular bundle it is safer than an open approach to the ganglion. Resection of the ganglion can result in immediate improvement of the symptoms, however, in case of chronic disease, the affected nerve may take some time to recover. If the ganglion cannot be resected it may still form back on its own once the intraarticular damage was addressed. In case of excess production of synovial fluid on the base of rheumatic disease, injections with corticosteroids might be a good option [1] [2].

There are further studies necessary to evaluate typical symptoms corresponding to various locations of the cysts, for further differentiation from lumbar or vascular pathologies. Furthermore, optimal arthroscopic approaches for draining the cysts need to be identified.

\section{Conflicts of Interest}

The authors declare no conflicts of interest regarding the publication of this paper.

\section{References}

[1] Angelini, A., Zanotti, G., Berizzi, A., Staffa, G., Piccinini, E. and Ruggieri, P. (2017) Synovial Cysts of the Hip. Acta Biomedica, 88, 483-490.

[2] Yukata, K., Nakai, S., Goto, T., et al. (2015) Cystic Lesion around the Hip Joint. World Journal of Orthopedics, 6, 688-704. https://doi.org/10.5312/wjo.v6.i9.688

[3] Botchu, R., Esler, C.N., Lloyd, D.M. and Rennie, W.J. (2013) Ganglia Arising from the Transverse Acetabular Ligament: A Report of Two Cases. Journal of Orthopaedic Surgery (Hong Kong), 21, 380-382. https://doi.org/10.1177/230949901302100324

[4] Chandler, S.K. (1934) The Iliopsoas Bursa in Man. The Anatomical Record, 58, 235-240. https://doi.org/10.1002/ar.1090580304

[5] Robinson, P., White, L.M., Agur, A., Wunder, J., Bell, R.S. and Robinson, R. (2003) Obturator Externus Bursa-Origin and MR Features of Pathology. 
[6] Magee, T. and Hinson, G. (2000) Association of Paralabral Cysts with Acetabular Disorders. American Journal of Roentgenology, 174, 1381-1384. https://doi.org/10.2214/ajr.174.5.1741381

[7] Magerkurth, O., Jacobson, J.A., Girish, G., Brigido, M.K., Bedi, A. and Fessell, D. (2012) Paralabral Cysts in the Hip Joint: Findings at MR Arthrography. Skeletal Radiology, 41, 1279-1285. https://doi.org/10.1007/s00256-012-1395-4

[8] Jo, S., Hooke, A.W., An, K.N., Trousdale, R.T. and Sierra, R.J. (2018) Contribution of the Ligamentum Teres to Hip Stability in the Presence of an Intact Capsule: A Cadaveric Study. Arthroscopy: The Journal of Arthroscopic \& Related Surgery, 34, 1480-1487. https://doi.org/10.1016/j.arthro.2017.12.002

[9] Kraeutler, M.J., Garabekyan, T., Pascual-Garrido, C. and Mei-Dan, O. (2016) Ligamentum Teres Tendinopathy and Tears. Muscle, Ligaments and Tendons Journal, 6, 337-342. https://doi.org/10.32098/mltj.03.2016.09

[10] Martin, R.L., McDonough, C., Enseki, K., Kohreiser, D. and Kivlan, B.R. (2019) Clinical Relevance of the Ligamentum Teres: A Literature Review. International Journal of Sports Physical Therapy, 14, 459-467. https://doi.org/10.26603/ijspt20190459

[11] Rosinsky, P.J., Shapira, J., Lall, A.C. and Domb, B.G. (2020) All about the Ligamentum Teres: From Biomechanical Role to Surgical Reconstruction. The Journal of the American Academy of Orthopaedic Surgeons, 28, E328-E339. https://doi.org/10.5435/JAAOS-D-19-00352

[12] Park, J., Kang, Y., Ahn, J.M., Lee, E., Lee, J.W. and Kang, H.S. (2019) Non-Traumatic Ligamentum Teres Tears: Association with MRI Morphometry of the Hip. Acta Radiologica, 60, 615-622. https://doi.org/10.1177/0284185118788897

[13] Schmitz, M.R., Campbell, S.E., Fajardo, R.S. and Kadrmas, W.R. (2012) Identification of Acetabular Labral Pathological Changes in Asymptomatic Volunteers Using Optimized, Noncontrast 1.5-T Magnetic Resonance Imaging. The American Journal of Sports Medicine, 40, 1337-1341. https://doi.org/10.1177/0363546512439991

[14] Register, B., Pennock, A.T., Ho, C.P., Strickland, C.D., Lawand, A. and Philippon, M.J. (2012) Prevalence of Abnormal Hip Findings in Asymptomatic Participants: A Prospective, Blinded Study. The American Journal of Sports Medicine, 40, 2720-2724. https://doi.org/10.1177/0363546512462124

[15] Stuplich, M., Hottinger, A.F., Stoupis, C. and Sturzenegger, M. (2005) Combined Femoral and Obturator Neuropathy Caused by Synovial Cyst of the Hip. Muscle and Nerve, 32, 552-554. https://doi.org/10.1002/mus.20364

[16] Yukata, K., Arai, K., Yoshizumi, Y., Tamano, K., Imada, K. and Nakaima, N. (2005) Obturator Neuropathy Caused by an Acetabular Labral Cyst: MRI Findings. AJR American Journal of Roentgenology, 184, 112-114. https://doi.org/10.2214/ajr.184.3_supplement.0184s112

[17] Bachar, A.I., Amar, E., Efrima, B., Kollander, Y., Rath, E. and Volaski, H. (2018) Hip Arthroscopy as a Treatment for Obturator Neuropathy Secondary to Intra-Pelvic Ganglion: A Case Report. Journal of Hip Preservation Surgery, 5, 319-322. https://doi.org/10.1093/jhps/hny023

[18] Anderson, S., Ryan, J. and Baria, M.R. (2020) Obturator Internus Synovial Cyst. American Journal of Physical Medicine \& Rehabilitation, 99, e134-e135.

[19] Vidoni, A., Sankara, S.T.V., Ramana, V. and Botchu, R. (2019) Ganglion Cyst Arising from the Transverse Acetabular Ligament (TAL): A Rare Cause of Entrapment of the Anterior Branch of the Obturator Nerve. Case Report and Review of the Literature. Skeletal Radiology, 48, 163-165. https://doi.org/10.1007/s00256-018-2992-7 
[20] Kim, S.H., Seok, H., Lee, S.Y. and Park, S.W. (2014) Acetabular Paralabral Cyst as a Rare Cause of Obturator Neuropathy: A Case Report. Annals of Rehabilitation Medicine, 38, 427-432. https://doi.org/10.5535/arm.2014.38.3.427

[21] Jitpun, E., Howe, B., Matthew, M., Amrami, K.K., Trousdale, R.T. and Spinner, R.J. (2019) Obturator Intraneural Ganglion Cysts: Joint Connected and Underdiagnosed. World Neurosurgery, 126, e259-e269. https://doi.org/10.1016/j.wneu.2019.02.029

[22] Campeas, S. and Rafii, M. (2002) Pelvic Presentation of a Hip Joint Ganglion: A Case Report. Bulletin of the NYU Hospital for Joint Diseases, 61, 89-92.

[23] Pergaminelis, N., Renouf, J., Fary, C., Tirosh, O. and Tran, P. (2017) Outcomes of Arthroscopic Debridement of Isolated Ligamentum Teres Tears Using the iHOT-33. BMC Musculoskeletal Disorders, 18, 4-9.

https://doi.org/10.1186/s12891-017-1905-6

[24] Colasanti, M., Sapienza, P., Moroni, E., Mosiello, G., Postacchini, F. and di Marzo, L. (2006) An Unusual Case of Synovial Cyst of the Hip Joint Presenting as Femoral Vein Compression and Severe Lower Limb Edema. European Journal of Vascular and Endovascular Surgery, 32, 468-470. https://doi.org/10.1016/j.ejvs.2006.05.012

[25] Briem, T., Haemmerle, G., Kramers-de Quervain, I. and Leunig, M. (2016) Synovial Ganglion of the Hip as a Rare Cause of L5 Radiculopathy: A Case Report. JBJS Case Connect, 6, e59. https://doi.org/10.2106/JBJS.CC.15.00234

[26] Hafezi-nejad, N., Vaidya, D. and Eng, J. (2018) MR Arthrography-A Sytematic Review an Meta-Analysis. American Journal of Roentgenology, 211, 52-63. 